\title{
Splice-site variant in ACSL5: a marker promoting opposing effect on cell viability and protein expression
}

\author{
Iván Pérez-Núñez ${ }^{1}$ Mohamad Karaky ${ }^{1}$ - María Fedetz ${ }^{1}$ - Cristina Barrionuevo ${ }^{1}$ Guillermo Izquierdo ${ }^{2}$ \\ Fuencisla Matesanz ${ }^{1} \cdot$ Antonio Alcina $^{1}$
}

Received: 11 June 2018 / Revised: 21 February 2019 / Accepted: 6 April 2019 / Published online: 3 May 2019

(c) The Author(s) 2019. This article is published with open access

\begin{abstract}
Long-chain Acyl-CoA synthetases (ACSLs) activate fatty acids (FAs) by thioesterification with Coenzyme A (CoA), generating FA-CoAs. These products are essential for lipid metabolism and carcinogenesis. In previous study, we identified an intronic variant rs2256368:A $>\mathrm{G}$, whose $\mathrm{G}$ allele promotes exon 20 skipping in up to $43 \%$ of ACSL5 transcripts but its functional relevance is unclear. Here, we compared the expression of splice (Spl) and nonsplice (NSpl) ACSL5 variants and the effect on cell viability under culture conditions that force cells to metabolize fatty acids. We found that lymphoblastoid cell lines from 1000 Genomes Project, bearing Spl genotypes, showed a reduced expression of total ACSL5 protein due to an inefficient translation of the Spl RNA. These cells impaired growth in cultures with phorbol myristate acetate-ionomycin (PMA-Io) or medium deprived of glucose, while production of reactive oxygen species increased in PMA-Io. Specific ACSL5-isoform transfection in HEK239T (kidney), U87 (astroglioma), and HOG (oligodendrocyte) cells showed the Spl protein to be the causal factor of cell-growth inhibition, despite its reduced protein expression. Our findings indicate that the variant rs2256368:A $>\mathrm{G}$ can predict a growth inhibitory activity, caused by the Spl isoform of ACSL5 protein, opposed to the activity of the NSpl. Deep understanding of its functioning might have application in metabolic diseases and cancer.
\end{abstract}

\section{Introduction}

Long-chain acyl-CoA synthetases (ACSLs) activate fatty acids (FAs: C12-C20), by thioesterification with Coenzyme A

These authors contributed equally: Iván Pérez-Núñez,

Mohamad Karaky

Supplementary information The online version of this article (https:// doi.org/10.1038/s41431-019-0414-5) contains supplementary material, which is available to authorized users.

\footnotetext{
Fuencisla Matesanz

lindo@ipb.csic.es

$\bowtie$ Antonio Alcina

pulgoso@ipb.csic.es

1 Department of Cell Biology and Immunology, Instituto de Parasitología y Biomedicina "López Neyra" (IPBLN), Consejo Superior de Investigaciones Científicas (CSIC), 18016 Granada, Spain

2 Unidad de Esclerosis Múltiple, Hospital Universitario Virgen Macarena, 41009 Sevilla, Spain
}

(CoA), generating FA-CoAs. These products are the initial step in fatty acid metabolism. Once activated, they are involved in cell growth, differentiation, and energy regulation [1-5]. Fatty acids physiologically regulate ACSL expression, but cancer cells could hijack certain involved regulatory mechanisms to deregulate ACSLs. This deregulation is also associated with poor survival in patients with cancer [6].

ACSL5 is implicated in several types of cancers and has a potential prognostic value [6-12]. Unlike other ACSLs, ACSL5 is downregulated in colorectal carcinomas [13, 14], breast [10, 15], bladder [15], and pancreas [12]. However, ACSL5 is overexpressed in glioma [16] and considered a potential therapeutic target [17-19]. In addition, the fibroblast growth factor receptor 2 (FGFR2) ACSL5 chimera RNA rendered clinical gastric cancer cells resistant to treatment with FGFR inhibitors [6, 20].

Excess fatty acid metabolism has increasingly been found to be associated with metabolic disorders and carcinogenesis [6]. Genetic variants in ACSL5 may provide a linkage between the higher prevalence of cancer in obesity. For instance, ACSL5 rs2419621 T allele carriers are more responsive to lifestyle interventions partly due to an 
increase in the short ACSL5 protein isoform, increasing cellular, tissue, and whole-body fatty acid utilization [21]. Also, ACSL5 rs7903146 is one of the most strongly associated type 2 diabetes loci and resides within a regulatory region of an ACSL5 promoter [22].

The $\mathrm{G}$ allele of the rs2256368:A>G variant, located at intron 20 of ACSL5 gene was identify as the cause of exon 20 skipping in up to $43 \%$ of transcript molecules, using expression quantitative trait loci strategy [23]. The present work characterizes the functional effects promoted by this splice (Spl) variant. With this end, we compared the expression of $\mathrm{Spl}$ and nonsplice (NSpl) ACSL5 isoforms and the effect on cell viability in lymphoblastoid cell lines (LCLs) from 1000 Genomes Project and in other cell lines from different tissues.

\section{Materials and methods}

\section{Nomenclature and database submission}

Rs2256368:A $>\mathrm{G}$ variant was submitted to the LOVD 3.0/ shared with the submission ID \#60251 (http://databases. lovd.nl/shared/view/ACSL5). This variant (hg19 chr10: g. $114186624 \mathrm{G}>\mathrm{A})$ is located in the ACSL5 gene (NM_016234.3; c.2079+7G $>A$ ). The $G$ allele promotes exon 20 skipping in ACSL5 transcripts (r.2008_2079del) producing spliced (Spl) ACSL5- $\Delta 20$ RNAs [23].

\section{Selection of lymphoblastoid cell lines (LCLs)}

Twelve LCLs from HapMap and 1000 Genomes projects, were purchased from Coriell cell repository (Coriell Institute for Medical Research, Camden, NJ, USA). These 12 lines represented the three genotypes of rs2256368:A>G variant: genotype GG in cell lines HG00134, HG00326, HG01048, HG01383; genotype AG in lines GM12004, GM12044, GM12144, GM12717; and genotype AA in lines NA12006, NA11994, NA12043, NA11993. All cells were cultured in RPMI+10\% FCS as previously described [24].

\section{Relative quantification of RNA in LCLs}

ACSL5 RNA concentrations in LCLs were measured by real-time reverse transcription (RT) qPCR, normalizing the results to UBE2D2 RNA levels as reported in an earlier study, using the $2 \mathrm{E}$ deltaCt (deltaCt $=\mathrm{Ct}$ sample-Ct reference) method [25]. The primer sequences were (key: forward-Fw; reverse-Rv; E, exon; 5'-3' direction): UBE2D2 Fw- CAATTCCGAAGAGAATCCACAAGGAATTG and Rv- GTGTTCCAACAGGACCTGCTGAACAC; nonspliced (NSpl) E20 ACSL5 (using a bridge E19-E20 to E21) Fw-CCAAGTTGTAAGGGAAGCCA and Rv-GCTG
TCAATTTGGGTCCGAA; Spl E20 ACSL5 (using bridge E19-E21 to E21) Fw-ACTGTGCCAAAACCAAGTCA and Rv- TGTGCTCATACAGGCTGTCA.

\section{Cell extracts}

Total cell extracts were acquired using RIPA buffer (50 mMTris- $\mathrm{HCl} \mathrm{pH} 7.4,150 \mathrm{mMNaCl}, 1 \mathrm{mM}$ EDTA, $0.5 \%$ Na-deoxycholates, $0.1 \%$ SDS) plus antiproteases from the Halt Protease Inhibitor Single-Use Cocktail (Pierce, Rockford, IL, USA). Mitochondrial extracts were isolated from LCL NA12006E representing genotype AA, GM12004D representing genotype AG, and HG00134 representing genotype GG, using the Mitochondria Isolation Kit for mammalian cells (Pierce) following the manufacturer's instructions and using RIPA buffer for protein extraction.

\section{Western blots}

Cell extracts in RIPA buffer were processed for protein separation by sodium-dodecyl sulfate-7\% polyacrylamide gel electrophoresis (SDS-PAGE) under reducing conditions and transferred to Immobilon-P transfer membranes (Merck-Millipore Ltd., Cork, Ireland). Blots were incubated with different antibodies from Abcam (Abcam plc, Cambridge, UK). As primary antibodies we used anti-ACSL5 (ab57210) and anti-V5 tag (ab27671); as a secondary antibody, a horseradish peroxidase (HRP)-labeled anti-mouse IgG (ab97023) was used. As a loading control, we used an anti-beta Actin-HRP- labeled antibody (Ab8226) following conditions indicated by the manufactures. Protein bands were detected by an enhanced chemiluminescent substrate for detection of HRP using Pierce ECL Western Blotting Substrate (ThermoScientific, Rockford, IL, USA).

\section{ACSL5 protein quantification in LCLs}

Relative expression of ACSL5 protein was determined by band densitometry of the signal generated on a western blot film, using Bio-Rad's Image Lab Software (Bio-Rad Laboratories, Hercules, California, USA) as indicated in Supplementary Methods. The band densitometry results were normalized twice: (1) against beta-actin; and (2) against the highest concentration of ACSL5 protein.

\section{Cell viability of LCLs}

The 12 LCLs were cultured at 2500 cells per well in 96-well microtiter plates in three different culture conditions: (i) control cultures (CTL) with the usual growing media indicated above; (ii) cultures treated with phorbol myristate acetate-ionomycin (PMA-Io) $[50 \mathrm{ng} / \mathrm{ml}$ of phorbol 
12-myristate 13-acetate (PMA) plus $10 \mathrm{ng} / \mathrm{ml}$ Ionomycin (Io) [Sigma Aldrich, Madrid, Spain]); and (iii) glucosedepleted culture media (no glucose) plus $10 \%$ FCS. Growth measurements were performed at 6,24 , and $48 \mathrm{~h}$ of culture. Cell viability was determined using the Cell-Titer-Glo Luminescent Cell Viability Kit (Promega, Madison, WI, USA). All readings were first normalized with the readings at $6 \mathrm{~h}$ (growth index) and then, the relative growth of each LCL in PMA-Io-treated media or in glucose depleted cultures, were normalized to the growth index of the CTL cultures (relative growth index). Each time point shows the mean growth of four LCLs with the same genotype, and the standard error of mean (+/-SEM). Each experiment was performed five times for each condition in 12 replicates.

\section{ROS production in LCLs}

ROS production was measured in the 12 LCLs cultured in media treated with PMA-Io, seeded at 5000 cells per well in 96-well microplates for $0.5,2,4$, and $6 \mathrm{~h}$, using the Cellular Reactive Oxygen Species Detection Assay Kit (Abcam, ab113851), following the manufacturer's instructions. This kit uses the cell permeant reagent $2^{\prime}, 7^{\prime}$-dichlorofluorescin diacetate (DCFDA) to measure ROS activity within the cell. The resulting oxidation of DCFDA by intracellular ROS was detected by fluorescence spectroscopy with an Infiniti Lumi Microplate Reader (Tecan, Mannedorf, Switzerland) with maximum excitation and emission spectra of 495 and $529 \mathrm{~nm}$, respectively.

\section{Cloning of ACSL5 isoforms}

RNA from one LCL bearing rs2256368 GG genotype, which produced about $60 \% \mathrm{NSpl}$ and $40 \%$ Spl ACSL5 transcripts, was reverse-transcribed (RT) [23]. The cDNA was amplified by PCR using the following primers (in bold is the linker for directional cloning): Fw-CACCAGCACGTTAGAAAGCCTGAC and Rv-ATCCTGGATGTGCTCATACA, with the Q5 High-Fidelity DNA Polymerase Kit (New England BioLab, Ipswich, MA, USA), in the following PCR conditions: 1 cycle of $94^{\circ} \mathrm{C}$ for $3 \mathrm{~min} ; 34$ cycles of $94{ }^{\circ} \mathrm{C}$ for $30 \mathrm{~s} ; 60^{\circ} \mathrm{C}$ for $30 \mathrm{~s} ; 72^{\circ} \mathrm{C}$ for $2 \mathrm{~min}$. The PCR products were cloned into the expression plasmid pcDNA6.2/GW/D-TOPO (Invitrogen, Carlsbad, CA, USA). The resulting plasmid inserts were amplified by PCR from E19 of ACSL5 to the V5 tag sequence in the plasmid using primers: ACSL5 exon (E)19 Fw-CTTCCCTCATTTGCAGCCAA and V5 Rv- CCTAACCCTCTCCTCGGTCT. The PCR conditions were: 1 cycle of $94^{\circ} \mathrm{C}$ for $3 \mathrm{~min}$, followed by 34 cycles of $94{ }^{\circ} \mathrm{C}$ for $20 \mathrm{~s}, 60^{\circ} \mathrm{C}$ for $20 \mathrm{~s}$, plus $72{ }^{\circ} \mathrm{C}$ for $1 \mathrm{~min}$. The plasmids DNAs were validated by Sanger sequencing and purified with an EndoFree Plasmid Maxi Kit (QIAGEN $\mathrm{GmbH}$, Hilden, Germany) for transfection experiments.

\section{Transfection of different cell lines with ACSL5 isoforms}

HEK293 (human embryonic kidney [epithelial]), U87MG (human brain [astroglioma]), and HOG (human oligodendrocyte) [26] cell lines were cultured in DMEM medium (low glucose for HEK) supplemented with $15 \%$ fetal calf serum and $100 \mathrm{U} / \mathrm{ml}$ penicillin/streptomycin (all from Gibco, Invitrogen. Carlsbad, CA, USA). In all $0.5 \times 10^{6}$ cells/well were seeded on a six-well plate, and transfected with the different recombinant plasmids using lipofectamine LTX reagent (Invitrogen) following the manufacturer's instructions. The expression of ACSL5 mRNA and ACSL5 protein was analyzed at different times post-transfection, contemplating $48 \mathrm{~h}$ post transfection as optimal (determined in preliminary experiments).

\section{Absolute RNA quantification by digital PCR}

RNA was purified from transfected HEK293 cells using the RNeasy Plus Mini Kit (Qiagen, Hilden, Germany) and reverse transcribed into cDNA using Superscript III First-Strand Synthesis SuperMix (Invitrogen) [26]. Expression of ACSL5V5 mRNA levels was subjected to absolute quantification by droplet digital qPCR (ddqPCR) using QX200 ddPCR Eva Green Supermix and QuantaSoft software (Bio-Rad).

The PCR amplification of the NSpl ACSL5-V5 construct was performed using a bridge primer that hybridizes with sequences in exons 19-20 (Fw-ACTGTGCCAAAAC CAAGTTG) and with the sequence of the V5 tag (RvAGACCGAGGAGAGGGTTAGG). The PCR amplification for the Spl ACSL5-V5 construct (ACSL5 $\Delta 20$-V5), was performed using a bridge primer that hybridizes with sequences in exons 19-21 (Fw-ACTGTGCCAAAACCAAGTCA), and with the sequence of the V5 tag (Rv), as indicated above.

\section{Cell viability of cell lines transfected with ACSL5 isoforms}

HEK293, U87MG, and HOG cell lines, transfected with the ACSL5 isoforms, were seeded at 2,500 cells per well in 96 -well microtiter plates, at $48 \mathrm{~h}$ post transfection. Cell viability was determined using the Cell-Titer-Glo Luminescent Cell Viability Kit (Promega, Madison, WI, USA) following manufacturer's instructions. Luminescence was measured using Infinite Lumi Microplate Reader (Tecan). The readings at 24 are indicated versus the readings at $6 \mathrm{~h}$. Each experiment was performed three times in 12 replicates. 


\section{Statistical analysis}

Statistical analysis was performed with RStudio software. Values are shown as mean \pm SEM and the number of independent experiments $(n)$ performed are indicated above. Data were analyzed by Pearson's correlation or ANOVA and Bonferroni post-hoc test. A value of $p<0.05$ was set as the limit of statistical significance.

\section{Results}

\section{ACSL5 isoforms}

ACSL5 expresses three major transcript isoforms, though only two full-length proteins have been usually observed [1]. Two of them have two in-frame AUG-translational initiators that produce isoforms of different length (Fig. 1a). The longer product (L) is initiated at the first AUG codon, AUG1, corresponding with variant 1 . A shorter product (S) is initiated at the downstream AUG2 (v3). A third variant is generated by alternative splicing of the penultimate exon of ACSL5, corresponding with variant $\mathrm{v} 2(\mathrm{Spl})$. This $\mathrm{Spl}$ isoform is promoted by the genetic variant rs2256368:A $>\mathrm{G}$ [23].

\section{ACSL5 isoforms expressed in LCLs}

In order to check the expression of major ACSL5 protein isoforms in LCLs, a western blot of extracts from cells expressing representative \% of Spl and NSpl RNAs, with an anti-ACSL5 antibody, was performed (Fig. 1b). Results showed similar band profiles in the three genotypes. However, the Spl-protein bands, with $3.7 \mathrm{kDa}$ less than the $\mathrm{L}$ and $\mathrm{S}$ isoforms, were not observed. GG-bearing cells, which expressed $40 \%$ of SplRNA, showed an important decrease in total ACSL5 protein.

\section{Expression analysis of RNA and protein isoforms versus genotypes}

For the purpose of finding an explanation to the previous observations, we carried out an expression analysis of RNA and protein isoform levels in $12 \mathrm{LCLs}$, four of each genotype (Fig. 2). For instance, the LCL no. 10, carrier of GG genotype, produced $43 \%$ of Spl RNA (Fig. 2a), but the Spl protein was not apparently detected (Fig. 2b). The correlation analysis showed that the increase of Spl RNA, and the G-allele doses, was linked to the decrease of total ACSL5 protein (Fig. 2c). The high determination coefficient $\left(r^{2}=0.69\right)$ suggested that the major part of the ACSL5 protein reduction corresponded to the increase of Spl RNA (Fig. 2d). All of these data suggested that the expression of Spl-ACSL5 protein was downregulated as compared to the NSpl isoforms.

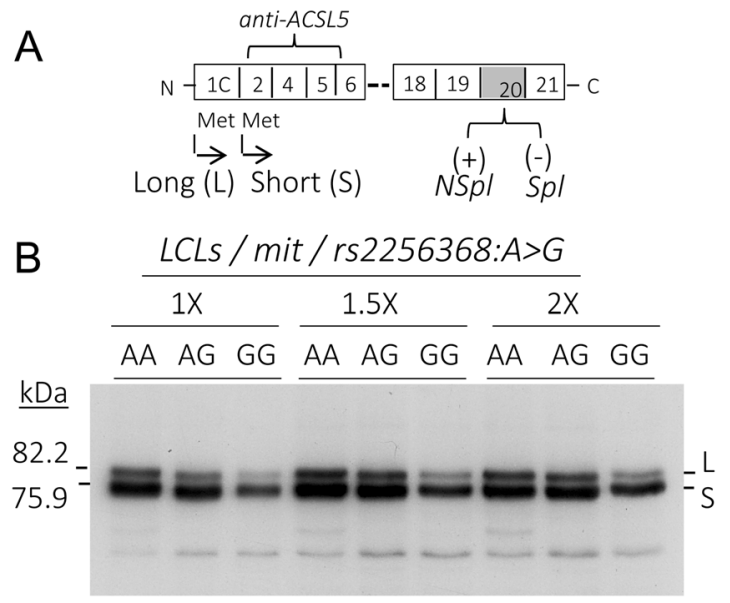

Fig. 1 Expression of ACSL5 protein isoforms in LCLs. a ACSL5 scheme representing the major protein isoforms. b Western blots of mitochondrial (mit) extracts from LCLs bearing the three rs2256368:A $>$ G genotypes (AA, AG, GG) loaded at different concentrations (1X, 1.5X and $2 \mathrm{X})$. The three LCLs expressed different proportions of Spl RNA respect to the NSpl one (AA- 1.8\%, AG$14 \%$, and GG-43\%). Molecular weights of ACSL5 L and S were calculated from the amino acid sequence

\section{Ectopic expression of Spl and NSpl isoforms}

To elucidate if the SplRNA was translated into protein, HEK293 cells were transfected with the Spl and NSpl cDNA constructs. The results showed that the both RNA isoforms, Spl and NSpl, were expressed similarly in the corresponding transfected cells (Fig. 3a). However, a dramatic decrease of Spl-protein levels was observed, without signs of differential degradation. Thus, these data suggested that the Spl-RNA was translated into protein in a very inefficient way, compared to the NSpl-isoform. In addition, these data explained why the Spl protein, endogenously expressed in LCLs bearing GG genotypes, was not detected.

\section{Cell viability of LCL bearing Spl and NSpl genotypes}

Given the implications of ACSL5 in mitochondrial functions, we measured the ATP content (correlating with cell growth) in the 12 LCLs bearing the three rs2256368: $\mathrm{A}>\mathrm{G}$ genotypes (Fig. 4), under mitochondrial-related stress conditions. Thus, cells were cultured in culture media with PMA-Io, stimulating lymphocyte cell growth [25] (Fig. 4a), which increased the mitochondrial function; or in culture media depleted of glucose, called "No glucose" in the figure (Fig. 4b), forcing cells to use exogenous long-chain fatty acids as energy source. Growth of each treated culture was referred to the growth at $6 \mathrm{~h}$ and then to the corresponding untreated culture (relative growth index). Cells bearing AA genotype, stimulated with PMA-Io, responded increasing growth more 
A
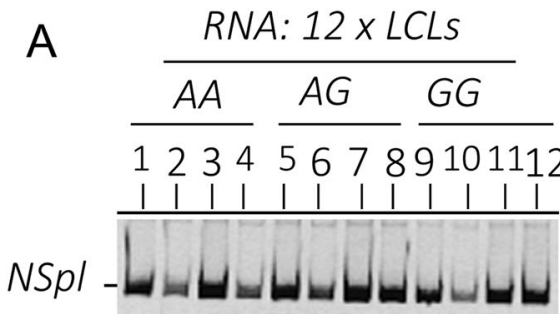

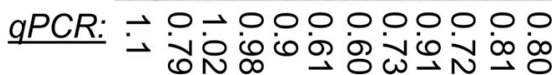

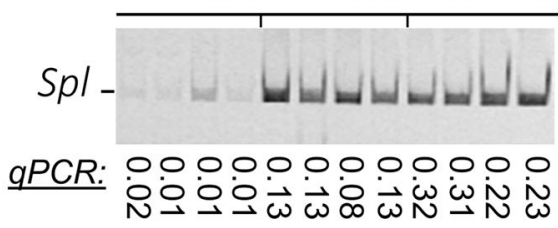

C

$\square$ Relative ACSL5 protein

$\square$ Ratio Spl vs. NSpl ACSL5 RNA

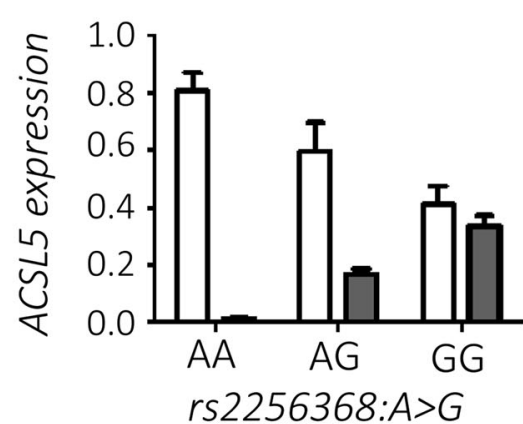

Fig. 2 Analysis of ACSL5 expression in LCLs. a Quantification of RNA isoforms in 12 LCLs. This panel has been previously published by Matesanz et al. [23], licensed under a Creative Commons Attribution-NonCommercial-ShareAlike 4.0 International License, http://creativecommons.org/licenses/by-nc-sa/4.0/. The panels show end-point PCR amplification visualized in polyacrylamide gel electrophoresis and, additionally, quantified by relative real-time qPCR: (top) Nonspliced ACSL5 transcripts (NSpl); (bottom) spliced ACSL5 transcripts (Spl). b Western blots with an anti-ACSL5 antibody of the

than the untreated cells and were not affected by absence of glucose within $48 \mathrm{~h}$ of culture, compared with standard medium with glucose and without PMA-Io. By contrary, cells bearing AG or GG genotypes were significantly affected, reducing the growth under PMA-Io stimulation, and in cultures depleted of glucose, respect to the control cultures. These findings suggested an association of the impaired cell growth capability with the $G$ allele carriers under mitochondrial stress. Thus, expression of the $\mathrm{SpL}$ protein and/or decrease of total ACSL5 protein were the two variables associated with these growth effects.
B

Protein: $12 \times L C L S$

$A A \quad A G \quad G G$

123456789101112
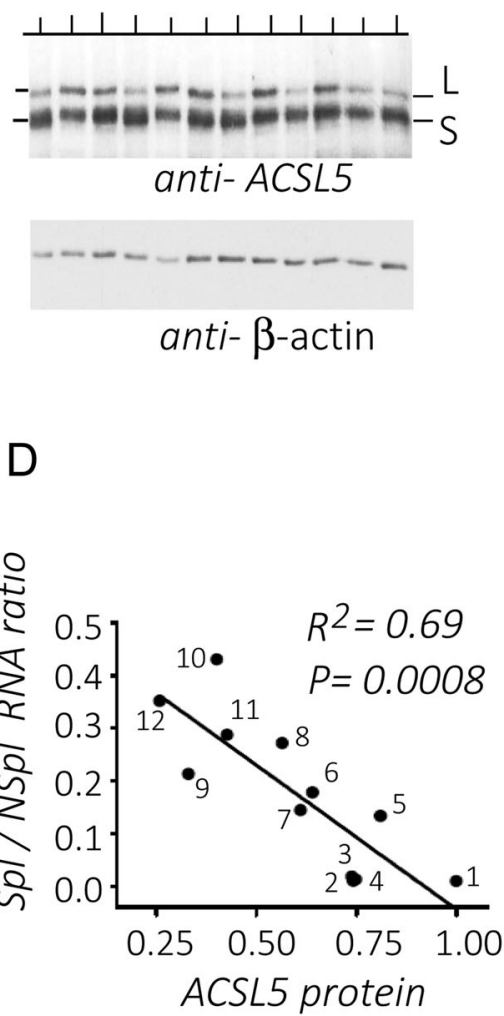

same LCLs as in the RNA panel. An anti-beta-actin HRP-labeled antibody was used as a loading control for relative protein quantification ("Materials and methods" and Supplementary Material S1). c Plot representing relative expression levels of ACSL5 protein $(\mathrm{L}+\mathrm{S})$ (white bars) and ratio of Spl versus NSpl RNAs (dark bars). d Correlation plot of Spl to NSpl RNA ratios versus relative ACSL5 protein levels. Pearson correlation was used to estimate determination coefficient $\left(R^{2}\right)$ and $P$-value

\section{Reactive oxygen species}

To assess whether the impaired cell growth of LCLs bearing the $\mathrm{G}$ allele was related with the oxidative stress response, cells were stimulated with PMA-Io up to $6 \mathrm{~h}$. Treatment with PMA-Io resulted in ROS production, which was always higher in cells expressing the Spl variant (GG bearing cells). The results were normalized to the positive control treatment, using tert-Butyl hydroperoxide (Fig. 4c). All these data suggested that the cells containing the rs2256368 G allele were negatively affected when mitochondrial-related stressors were present. 

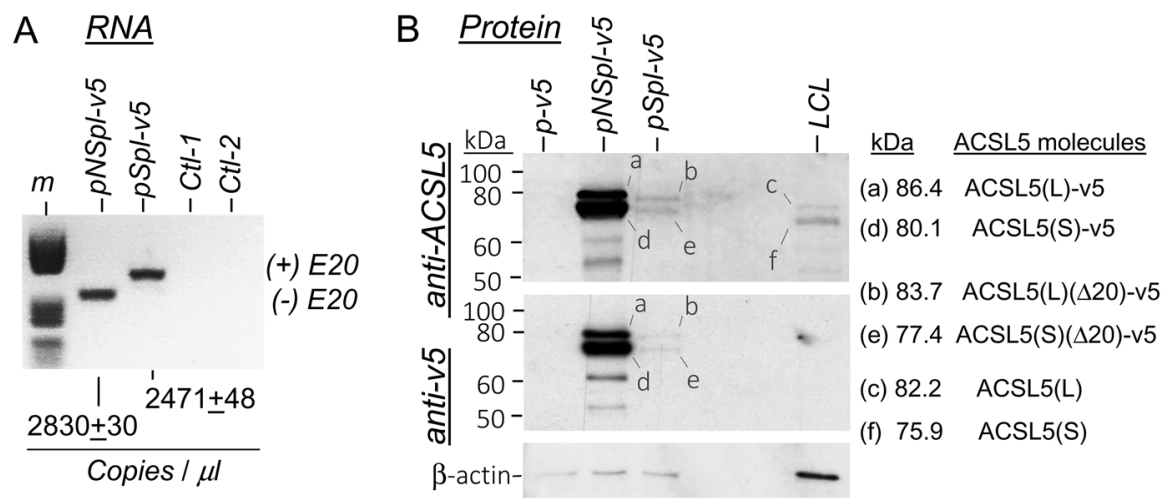

Fig. 3 Expression analysis of $\mathrm{Spl}$ and $\mathrm{NSpl}$ constructs in transfected HEK293 cells. a Droplet digital PCR quantification of RNA expression from the spliced $(\mathrm{pSpl})$ and nonspliced (pNSpl) cDNAs, visualized in agarose-gel electrophoresis. Ctl-1 and Ctl-2 were PCR negative controls (RT performed without RT enzyme) for each isoform. Lane $m$ was loaded with a DNA size marker mix (Marker V from Roche). b ACSL5 protein expression analysed by western blots of extracts from the transfected cells. Lane LCL is a positive control extract containing endogenous ACSL5 from one LCL, for comparison with the recombinant constructs from transfected cells. Lane $\mathrm{kDa}$ represents the position of molecular size markers. Letters and lines inside the blots are explained on the right site, representing the protein sequence-calculated molecular weights of the different ACSL5 molecules detected
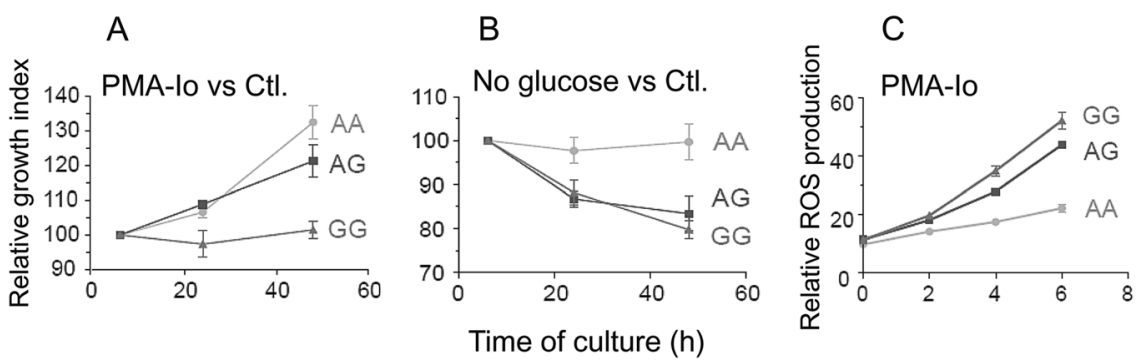

Fig. 4 Relative growth index of 12 LCLs, four of each rs2256368: $\mathrm{A}>\mathrm{G}$ genotype. Cells were cultured under conditions that force cells to use long-chain fatty acids and compared with the growth in control cultures. Student $t$-test was used to estimate difference between each genotype group. $P$-values $(P)$ at $48 \mathrm{~h}$ of culture were in a $P($ AA versus

\section{Identification of causal isoform of the growth inhibition}

Finally, to identify the ACSL5 isoform responsible for the growth inhibition, different cell lines (HEK293, U87, and HOG) were transfected with the different ACSL5 constructs (Fig. 5a). Thus, the cells transfected with the Spl construct (pSpl-V5) showed lower growth capability than the control cultures transfected with the plasmid (p-v5), as opposed to the growth capability of cells transfected with the NSpl construct (pNSpl-V5). Therefore, despite its low production, the Spl-V5 protein impaired cell growth in comparison to the NSpl-V5 and p-V5. On the other hand, ROS production (data not shown) was increased in all of these cell lines expressing recombinant Spl-V5 protein, but with no statistical significance, except U87 cells. This could happen because the induction times of ROS and the expression of the cDNA-V5 constructs did not coincide (data not shown).
$\mathrm{AG})=0.0487 ; \quad P(\mathrm{AG}$ versus $\mathrm{GG})=0.0013 ; \quad P(\mathrm{AA}$ versus $\mathrm{GG})$ $<0.0001$. b $P(\mathrm{AA}$ versus $\mathrm{AG})=0.0095 ; P(\mathrm{AG}$ versus $\mathrm{GG})=0.463$; $P($ AA versus $\mathrm{GG})=0.0003$. c Relative ROS production of the 12 LCLs in presence of PMA-Io

All these cells expressed the ACSL5 constructs in a similar way as shown in transfected HEK293 in Fig. 3b, but HOG cells expressed a higher ratio of Spl-V5 protein to NSpl-V5 (data not shown). Basal expression of RNA and protein was detected neither in HEK293 nor in U87. However, HOG cells expressed a minimal amount of RNA and protein (Fig. 5b, c).

\section{Discussion}

This study aimed at analyzing the functional role of the genetic variant rs 2256368: $A>G$ whose $G$ allele promotes exon 20 skipping in up to $43 \%$ of ACSL5 transcripts [23]. We found that the Spl RNAs were translated into Spl proteins very inefficiently, as compared with the translation levels of the NSpl isoforms.

The differential production of Spl and NSpl proteins was clearly observed in LCLs bearing GG genotype, expressing 
A

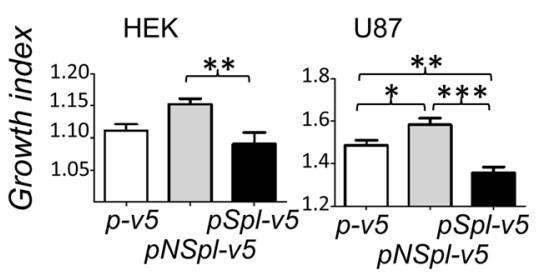

Transfected cell lines
B

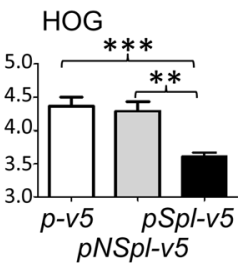

pNSpl-v5

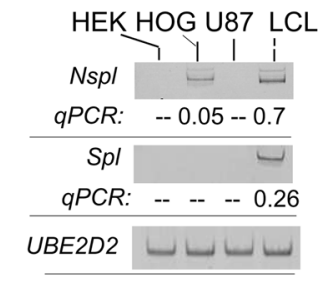

Basal RNA
C

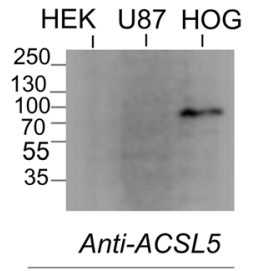

Basal protein
Fig. 5 Effect of ACSL5 isoforms on cell viability. a Growth index of cells transfected with control plasmid (p-V5), nonspliced (pNSpl-V5) and spliced (pSpl-V5) constructs. Statistical significance is indicated with asterisks: $* P<0.05$; $* * P<0.005 ; * * * P<0.0005$. b Basal level of ACSL5 RNA in nontransfected cells. One LCL is included to show the

both RNA isoforms, as well as in cell lines transfected with the two cDNA constructs. In this last case, the Splprotein expression was drastically reduced compared with the NSpl isoform, from similar number of corresponding mRNA molecules. Consequently, one or several mechanisms of translation regulation, that take place in the cell, might be affecting the production of the Spl protein.

It has been described microRNAs (miRNAs) that block the translation initiation without detectable changes in mRNA levels [27, 28]. For the more highly repressed mRNA translation, its destabilization explains most miRNAmediated repression [29]. Another possible mechanism could be based on the cell quality control, preventing synthesis of aberrant Spl proteins at the ribosome [30, 31]. Aberrant, misfolded, and mislocalized proteins are often toxic to cells and result in many human diseases. Although this mechanism could also affect the Spl protein, we did not observe evidence of specific differential degradation.

We showed that the Spl protein was responsible of the growth-inhibitory effect, despite its low protein expression. The evidence were obtained from LCLs expressing the Spl isoform, cultured in conditions that forced cells to use longchain fatty acids, as well as from cell lines transfected with the two ACSL5 isoforms. In both cases, the growthinhibitory effect caused by the $\mathrm{Spl}$ protein was opposed to the growth-promoting activity of the NSpl isoform, inhibiting cell growth below the level of control cells. These results suggested that the activity of the Spl protein had a certain toxic effect, which would be attenuated by inefficient translation of the Spl variant. Using a conceptual explanation from Battle et al. [32], it seems that this "detrimental" impact on cell growth is being attenuated or buffered by a large reduction of protein production [30, 32, 33]. In addition, our data also support that NSpl ACSL5 levels may be essential to reduce ROS-related stress, driving to a correct cell growth.

A detrimental alternative splicing that involves exon 7 skipping in the SP140 transcripts has also been reported endogenous expression of $\mathrm{Spl}$ and $\mathrm{NSpl}$ isoforms, as a positive control. These data were obtained by real time qPCR and the amplified samples were run in a polyacrylamide gel electrophoresis. UBE2D2 expression was used as a reference. c Basal level of ACSL5 protein expression performed by western blots of nontransfected cell lines

$[34,35]$. The genetic variant that promotes this alternative splicing is associated with multiple sclerosis, Crohn's disease, and chronic lymphocytic leukemia. However, what associates with the indicated diseases is the reduction of the $\mathrm{NSpl}$ protein expression because the Spl protein variant was not expressed.

Exon 20 skipped variant of ACSL5 protein ( $\mathrm{Spl}$ ) was studied for the first time by Gassler et al. [36]. They revealed that the ratio of the ACSL5-full length and the SplACSL5 protein increases along the crypt-villus axis of human small intestine. This fact was considered of functional relevance for apoptosis of senescent enterocytes at the villus tip and suggested to be involved in the pathogenesis of several intestinal disorders such as intestinal neoplasia [36]. It is unknown if the Spl ACSL5 isoform was promoted or not by the genetic variant analyzed in this study. Given that ACSL5 is highly expressed in human small intestinal mucosa, it would be possible that a small amount of the transcript is Spl even in the nonsplicing genotype AA.

The different allelic carriers of this genetic variant may produce large differences in the expression of the Spl transcripts (expression quantitative trait loci, eQTL) and protein isoforms (protein quantitative trait loci, pQTL), thereby it can increase phenotypic variability and the susceptibility to complex traits in human populations [30, 33, 37, 38]. Future association studies are necessary to find conditions in which this variant is involved.

As a marker, this genetic variant may be useful to predict the presence of an Spl RNA besides of the corresponding Spl protein. Its low expression level seems to attenuate its strong growth-inhibition effect. ACSL5 is generally decreased in those cancer types where ACSL1 and ACSL4 are upregulated [6], so it would be interesting to deepen our understanding of the mechanisms implicated in this alternative splicing, because it seems that between both isoforms there is a potential response against the two type of cancer cells, the ones that upregulate ACSL5 and the ones that downregulate it. All of these results may be of 
relevance in conditions involving lipid metabolism and carcinogenesis.

Acknowledgements This work was supported by the Agencia Estatal de Investigación del Ministerio de Economía, Industria y Competitividad of Spain [grant number SAF2016-80595 to AA, FM, and GI]; Junta de Andalucía- FEDER [grant number CTS2704 to FM]. Funders had no involvement in the study design, the collection, analysis and interpretation of the data, the writing of the report and in the decision to submit the paper for publication.

\section{Compliance with ethical standards}

Conflict of interest The authors declare that they have no conflict of interest.

Publisher's note: Springer Nature remains neutral with regard to jurisdictional claims in published maps and institutional affiliations.

Open Access This article is licensed under a Creative Commons Attribution 4.0 International License, which permits use, sharing, adaptation, distribution and reproduction in any medium or format, as long as you give appropriate credit to the original author(s) and the source, provide a link to the Creative Commons license, and indicate if changes were made. The images or other third party material in this article are included in the article's Creative Commons license, unless indicated otherwise in a credit line to the material. If material is not included in the article's Creative Commons license and your intended use is not permitted by statutory regulation or exceeds the permitted use, you will need to obtain permission directly from the copyright holder. To view a copy of this license, visit http://creativecommons. org/licenses/by/4.0/.

\section{References}

1. Soupene E, Kuypers FA. Mammalian long-chain acyl-CoA synthetases. Exp Biol Med . 2008;233:507-21.

2. Yan S, Yang XF, Liu HL, Fu N, Ouyang Y, Qing K. Long-chain acyl-CoA synthetase in fatty acid metabolism involved in liver and other diseases: an update. World J Gastroenterol. 2015;21: 3492-8.

3. Bowman TA, O'Keeffe KR, D'Aquila T, Yan QW, Griffin JD, Killion EA, et al. Acyl CoA synthetase 5 (ACSL5) ablation in mice increases energy expenditure and insulin sensitivity and delays fat absorption. Mol Metab. 2016;5:210-20.

4. Fujimoto Y, Itabe H, Kinoshita T, Homma KJ, Onoduka J, Mori $\mathrm{M}$, et al. Involvement of ACSL in local synthesis of neutral lipids in cytoplasmic lipid droplets in human hepatocyte HuH7. J Lipid Res. 2007;48:1280-92.

5. Mashek DG, McKenzie MA, Van Horn CG, Coleman RA. Rat long chain acyl-CoA synthetase 5 increases fatty acid uptake and partitioning to cellular triacylglycerol in McArdle-RH7777 cells. J Biol Chem. 2006;281:945-50.

6. Tang Y, Zhou J, Hooi SC, Jiang YM, Lu GD. Fatty acid activation in carcinogenesis and cancer development: essential roles of longchain acyl-CoA synthetases. Oncol Lett. 2018;16:1390-6.

7. Chen WC, Wang CY, Hung YH, Weng TY, Yen MC, Lai MD. Systematic analysis of gene expression alterations and clinical outcomes for long-chain acyl-Coenzyme A synthetase family in cancer. PLoS ONE. 2016;12:e0155660.

8. Klaus C, Schneider U, Hedberg C, Schütz AK, Bernhagen J, Waldmann $\mathrm{H}$, et al. Modulating effects of acyl-CoA synthetase 5-derived mitochondrial Wnt2B palmitoylation on intestinal Wnt activity. World J Gastroenterol. 2014;20:14855-64.

9. Klaus C, Jeon MK, Kaemmerer E, Gassler N. Intestinal acyl-CoA synthetase 5: activation of long chain fatty acids and behind. World J Gastroenterol. 2013;19:7369-73.

10. Yen MC, Kan JY, Hsieh CJ, Kuo PL, Hou MF, Hsu YL. Association of long chain acyl coenzyme A synthetase 5 expression in human breast cancer by estrogen receptor status and its clinical significance. Oncol Rep. 2017;37:3253-60.

11. Ma W, Li T, Wu S, Li J, Wang X, Li H. LOX and ACSL5 as potential relapse markers for pancreatic cancer patients. Cancer Biol Ther. 2019;3:1-12.

12. Li H, Wang X, Fang Y, Huo Z, Lu X, Zhan X, et al. Integrated expression profiles analysis reveals novel predictive biomarker in pancreatic ductal adenocarcinoma. Oncotarget. 2017;8:52571-83.

13. Gassler N, Schneider A, Kopitz J, Schnölzer M, Obermüller N, Kartenbeck J, et al. Impaired expression of acyl CoA synthetase 5 in epithelial tumors of the small intestine. Hum Pathol. 2003;34: $1048-52$.

14. Hartmann F, Sparla D, Tute E, Tamm M, Schneider U, Jeon MK, et al. Low acyl-CoA synthetase 5 expression in colorectal carcinomas is prognostic for early tumor recurrence. Pathol Res Pract. 2017;213:261-6.

15. Gaisa NT, Reinartz A, Schneider U, Klaus C, Heidenreich A, Jakse G, et al. Levels of acyl coenzyme A synthetase 5 in urothelial cells and corresponding neoplasias reflect cellular differentiation. Histol Histopathol. 2013;28:353-64.

16. Yamashita Y, Kumabe T, Cho YY, Watanabe M, Kawagishi J, Yoshimoto T, et al. Fatty acid induced glioma cell growth is mediated by the acyl CoA synthetase 5 gene located on chromosome $10 \mathrm{q} 25.1 \mathrm{q} 25.2$, a region frequently deleted in malignant gliomas. Oncogene. 2000;19:5919-25.

17. Mashima T, Sato S, Okabe S, Miyata S, Matsuura M, Sugimoto $\mathrm{Y}$, et al. Acyl-CoA synthetase as a cancer survival factor: its inhibition enhances the efficacy of etoposide. Cancer Sci. 2009; 100:1556-62.

18. Mashima T, Oh-hara T, Sato S, Mochizuki M, Sugimoto Y, Yamazaki $\mathrm{K}$, et al. p53-defective tumors with a functional apoptosome-mediated pathway: a new therapeutic target. J Natl Cancer Inst. 2005;97:765-77.

19. Mashima T, Sato S, Sugimoto Y, Tsuruo T, Seimiya H. Promotion of glioma cell survival by acyl-CoA synthetase 5 under extracellular acidosis conditions. Oncogene. 2009;28:9-19.

20. Kim SY, Ahn T, Bang H, Ham JS, Kim J, Kim ST, et al. Acquired resistance to LY2874455 in FGFR2 amplified gastric cancer through an emergence of novel FGFR2 ACSL5 fusion. Oncotarget. 2017;8:15014-22.

21. Rajkumar A, Liaghati A, Chan J, Lamothe G, Dent R, Doucet É, et al. ACSL5 genotype influence on fatty acid metabolism: a cellular, tissue, and whole-body study. Metabolism. 2018;83: 271-9.

22. Xia Q, Chesi A, Manduchi E, Johnston BT, Lu S, Leonard ME, et al. The type 2 diabetes presumed causal variant within TCF7L2 resides in an element that controls the expression of ACSL5. Diabetologia. 2016;59:2360-8.

23. Matesanz F, Fedetz M, Barrionuevo C, Karaky M, Catalá-Rabasa A, Potenciano V, et al. A splice variant in the ACSL5 gene relates migraine with fatty acid activation in mitochondria. Eur J Hum Genet. 2016;24:1572-77.

24. Karaky M, Alcina A, Fedetz M, Barrionuevo C, Potenciano V, Delgado $\mathrm{C}$, et al. The multiple sclerosis-associated regulatory variant rs10877013 affects expression of CYP27B1 and VDR under inflammatory or vitamin $D$ stimuli. Mult Scler. 2016;22:999-1006.

25. Catalá-Rabasa A, Ndagire D, Sabio JM, Fedetz M, Matesanz F, Alcina A. High ACSL5 transcript levels associate with systemic 
lupus erythematosus and apoptosis in jurkat $\mathrm{T}$ lymphocytes and peripheral blood cells. PLoS One. 2011;6:e28591.

26. Bello-Morales R, Crespillo AJ, Praena B, Tabarés E, Revilla Y, García E, et al. Role of proteolipid protein in HSV-1 Entry in oligodendrocytic cells. PLoS One. 2016;11:e0147885.

27. Baek D, Villén J, Shin C, Camargo FD, Gygi SP, Bartel DP. The impact of microRNAs on protein output. Nature. 2008;455:64-71.

28. Cottrell KA, Chaudhari HG, Cohen BA, Djuranovic S. PTRE-seq reveals mechanism and interactions of RNA binding proteins and miRNAs. Nat Commun. 2018;9:301.

29. Eichhorn SW, Guo H, McGeary SE, Rodriguez-Mias RA, Shin C, Baek D, et al. mRNA destabilization is the dominant effect of mammalian microRNAs by the time substantial repression ensues. Mol Cell. 2014;56:104-15.

30. Wu L, Candille SI, Choi Y, Xie D, Jiang L, Li-Pook-Than J, et al. Variation and genetic control of protein abundance in humans. Nature. 2013;499:79-82.

31. Karamyshev AL, Karamysheva ZN. Lost in Translation: ribosome-associated mRNA and protein quality controls. Front Genet. 2018;9:431.

32. Battle A, Khan Z, Wang SH, Mitrano A, Ford MJ, Pritchard JK, Gilad Y. Genomic variation. Impact of regulatory variation from RNA to protein. Science. 2015;347:664-7.
33. Manning KS, Cooper TA. The roles of RNA processing in translating genotype to phenotype. Nat Rev Mol Cell Biol. 2017;18:102-14.

34. Matesanz F, Potenciano V, Fedetz M, Ramos-Mozo P, Abad-Grau Mdel $\mathrm{M}$, et al. A functional variant that affects exon-skipping and protein expression of SP140 as genetic mechanism predisposing to multiple sclerosis. Hum Mol Genet. 2015; 24:5619-27.

35. Karaky M, Fedetz M, Potenciano V, Andrés-León E, Codina AE, Barrionuevo C, et al. SP140 regulates the expression of immunerelated genes associated with multiple sclerosis and other autoimmune diseases by NF- $\mathrm{B}$ inhibition. Hum Mol Genet. 2018;27:4012-40234.

36. Gassler N, Roth W, Funke B, Schneider A, Herzog F, Tischendorf $\mathrm{JJ}$, et al. Regulation of enterocyte apoptosis by acyl-CoA synthetase 5 splicing. Gastroenterology. 2007;133:587-98.

37. Park E, Pan Z, Zhang Z, Lin L, Xing Y. The expanding landscape of alternative splicing variation in human populations. Am J Hum Genet. 2018;102:11-26.

38. Zhang X, Joehanes R, Chen BH, Huan T, Ying S, Munson PJ, et al. Identification of common genetic variants controlling transcript isoform variation in human whole blood. Nat Genet. $2015 ; 47: 345-52$ 